\title{
Jet Physics at CDF
}

\author{
Sally Seidel ${ }^{1}$ for the CDF Collaboration
}

Department of Physics and Astronomy, University of New Mexico, Albuquerque, NM 87131, USA seidel@phys.unm.edu

\section{Jets at CDF}

Jets have been studied by the CDF Collaboration [1] as a means of searching for new particles and interactions, testing a variety of perturbative QCD predictions, and providing input for the global parton distribution function (PDF) fits. Unless otherwise indicated below, the jets were reconstructed using a cone algorithm [2] with cone radius $R=0.7$ from data taken at the Fermilab Tevatron collider in Run 2, 2001-2003, with $\sqrt{s}=1.96 \mathrm{TeV}$. Central jets, in the pseudorapidity range relative to fixed detector coordinates $0.1<|\eta|<0.7$, are used.

\section{The Inclusive Jet Cross Section versus Transverse Energy}

A measurement has been made of the inclusive jet cross section using $177 \mathrm{pb}^{-1}$ of data. This cross section, which probes a distance scale below $10^{-17} \mathrm{~cm}$, stimulated interest in obtaining improved precision on PDFs when initial Run 1 measurements [3] showed an excess of data over theoretical expectations at high transverse energy $\left(E_{\mathrm{T}}\right)$. The analysis uncouples the systematic shift in the cross section associated with the combined effects of energy mismeasurement and resolution limitation of the detector from the statistical uncertainty on the data. The data span an $E_{\mathrm{T}}$ range of $44-550 \mathrm{GeV}$, extending the upper limit from Run 1 by almost $150 \mathrm{GeV}$. The data are compared to next-toleading (NLO) QCD predictions using the CTEQ6.1 PDF set [4] and found to be in good agreement. Figure 1 shows the ratio of measured and predicted cross sections as a function of $E_{\mathrm{T}}$. 


\section{CDF Run II Preliminary}
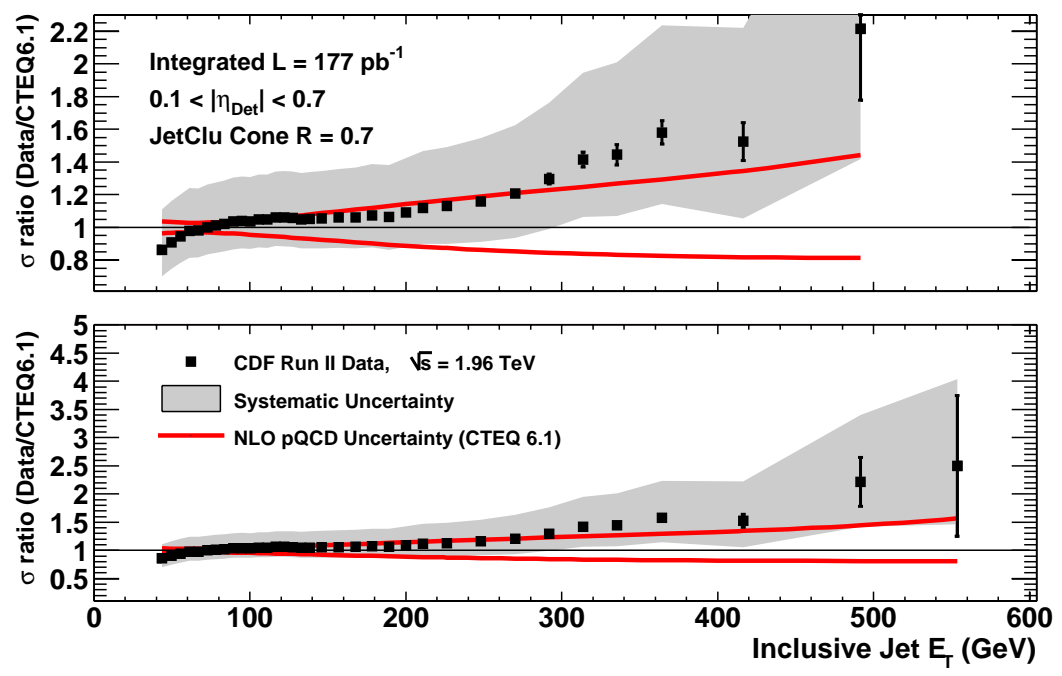

Fig. 1. The ratio of measured and predicted cross sections as a function of $E_{\mathrm{T}}$. The gray band indicates the combined experimental systematic uncertainty. The solid lines represent the uncertainty associated with choice of PDF.

\section{The Dijet Mass Spectrum}

The dijet mass spectrum has been examined for evidence of new particles. A general search has been made for narrow resonances, and a direct search has been made for several particle types. The dijet masses were fitted to a smooth background function plus a mass resonance to obtain $95 \%$ confidence level upper limits on the cross section for production of new particles, as a function of mass $M$. The upper limit is compared to cross section predictions for axigluons [5], flavor universal colorons [6], excited quarks [7], and $E_{6}$ diquarks [8]. At $95 \% \mathrm{CL}$, the search excludes a model of axigluons and colorons in the range $200<M<1130 \mathrm{GeV} / \mathrm{c}^{2}$, a model of excited quarks in the range $200<M<760 \mathrm{GeV} / \mathrm{c}^{2}$, and a model for $E_{6}$ diquarks in the range $280<M<420 \mathrm{GeV} / \mathrm{c}^{2}$. Figure 2 shows the production cross section times branching ratio upper limits for $75 \mathrm{pb}^{-1}$ of data.

\section{Jet Shapes}

Jets shapes may be characterized in differential and integrated form as $\rho(r)$ and $\Psi(r)$, respectively, where $r$ is a track's radial distance from the jet axis. The differential and integrated jet shapes are described by the average fraction of the jet's transverse energy that lies inside an annulus and 
Search for New Particles Decaying to Dijets

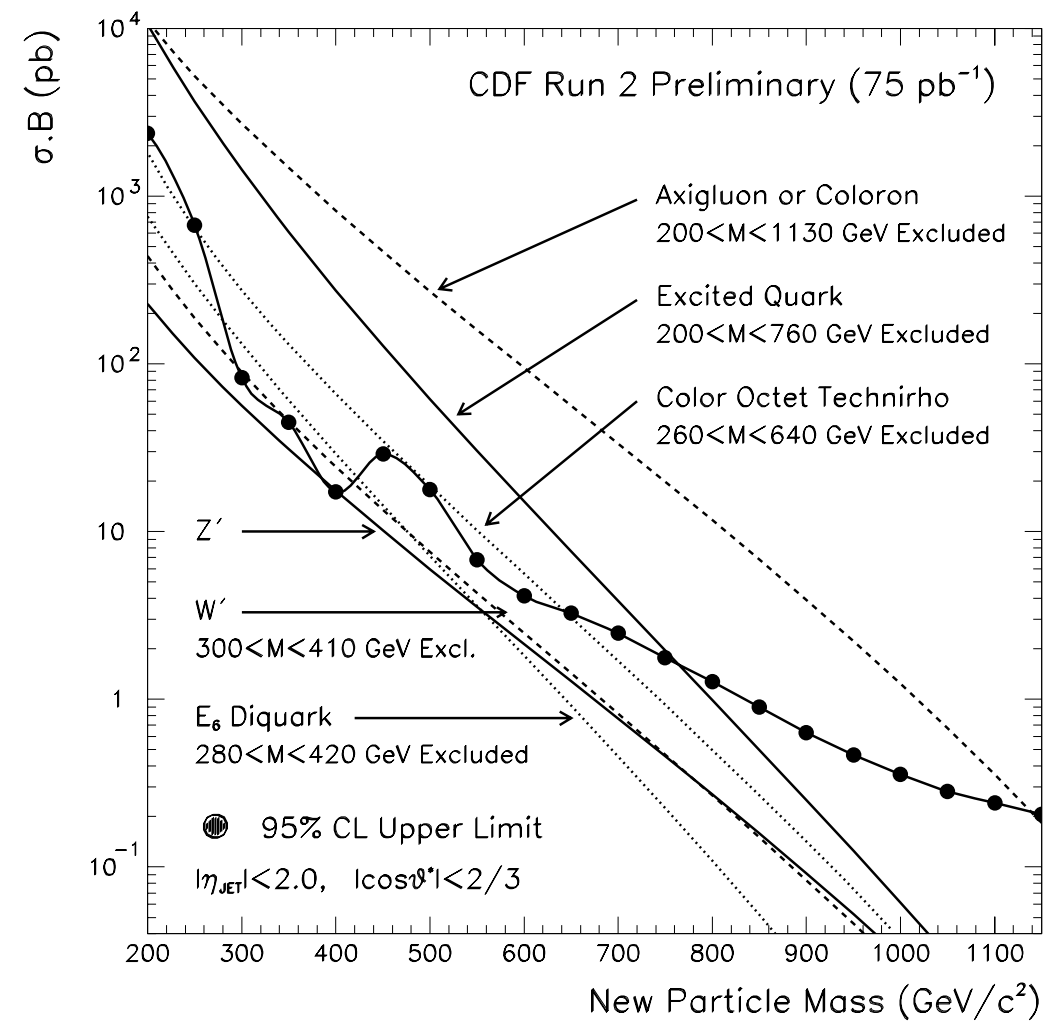

Fig. 2. The production cross section times branching ratio upper limits for new particles decaying to dijets, for $75 \mathrm{pb}^{-1}$ of data.

a cone, respectively, concentric with the jet cone axis in the plane defined by pseudorapidity $(\eta)$ and azimuthal angle $(\phi)$ relative to the detector. For an annulus of thickness $\Delta r$ and a cone of radius $R$, we define the differential distribution of a jet containing $N_{\text {jets }}$ jets as: $\rho(r)=$ $\frac{1}{N_{\mathrm{jet}}} \frac{1}{\Delta r} \sum_{\text {jets }} E_{\mathrm{T}}(r-\Delta r / 2, r+\Delta r / 2) / E_{\mathrm{T}}(0, R)$. We further define the integrated distribution by: $\Psi(r)=\frac{1}{N_{\mathrm{jet}}} \sum_{\text {jets }} E_{\mathrm{T}}(0, r) / E_{\mathrm{T}}(0, R)$. A total of $75 \mathrm{pb}^{-1}$ of data from calorimeter towers and from tracks in the central tracking chamber was examined, and the results were compared to leading order Monte Carlo predictions. Figures 3 and 4 show typical results for the differential and integrated distributions, respectively. One sees that HERWIG produces jets that are narrower than data, especially in the forward regions, but that the jet description improves with $E_{\mathrm{T}}$. PYTHIA describes jet shapes fairly well but produces jets narrower than the data in some kinematic regions, in particular 
at low $E_{\mathrm{T}}$. Figure 5 demonstrates, with an integrated jet shape measurement applied in three pseudorapidity regions for fixed cone opening angle $R=0.4$, that jets narrow as jet transverse energy increases.

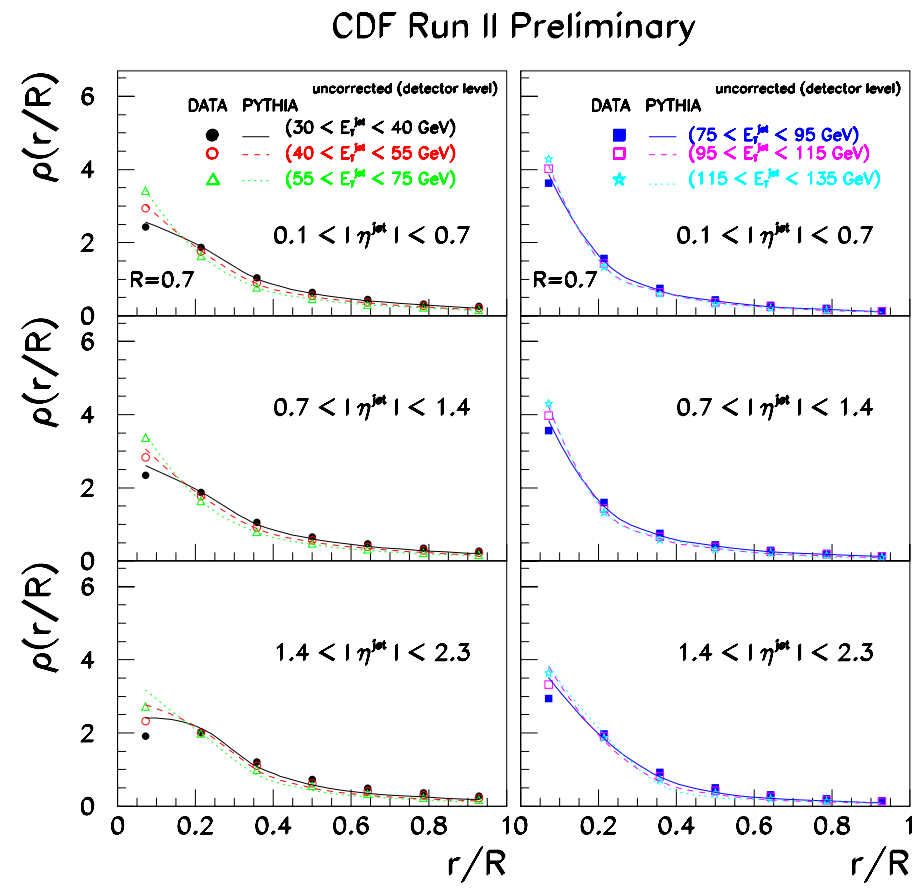

Fig. 3. The measured uncorrected differential jet shapes, $\rho(r)$, as computed using calorimetric information, in different regions of jet $E_{\mathrm{T}}$ and $\eta$, compared to Monte Carlo predictions.

\section{Jet Algorithms}

The cone and $k_{\mathrm{T}}$ [9] algorithms have been compared. The $k_{\mathrm{T}}$ algorithm, which successively merges pairs of nearby objects in order of decreasing $E_{T}$, uses a parameter $D$ to control the end of merging. The cone algorithm combines tracks into jets on the basis of their location relative to a cone of radius $R$ in $\eta-\phi$ space. For the purpose of comparing the algorithms, we set 


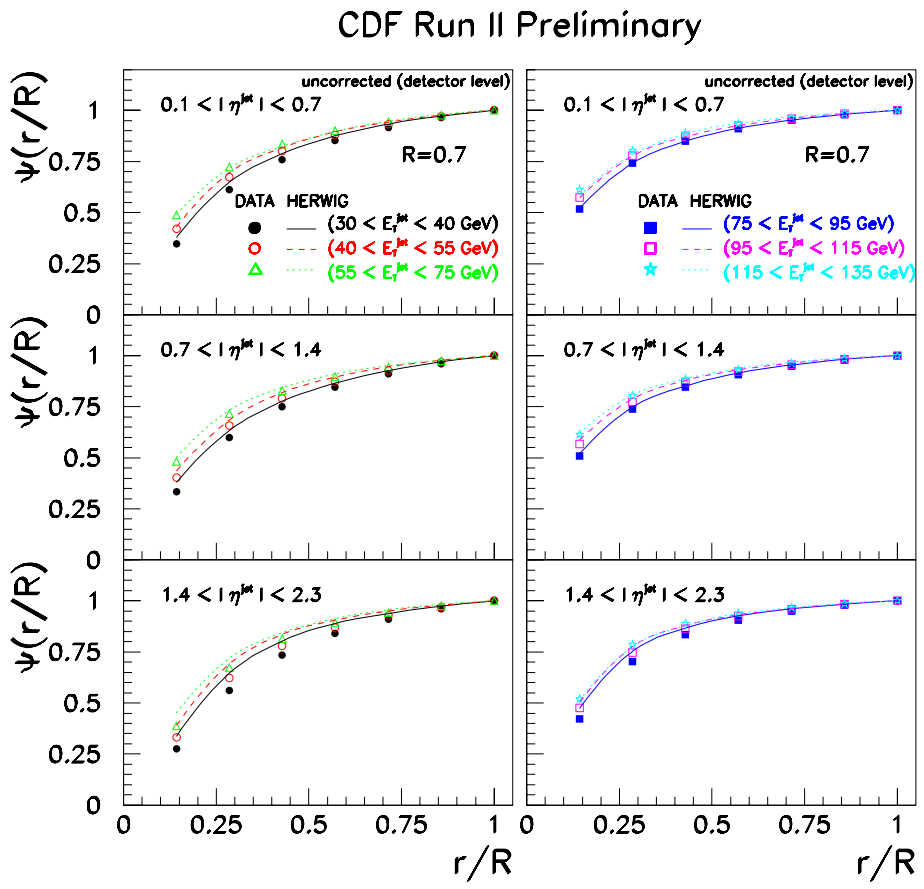

Fig. 4. The measured uncorrected integrated jet shapes, $\Psi(r)$, as computed using calorimetric information, in different regions of jet $E_{\mathrm{T}}$ and $\eta$, compared to Monte Carlo predictions.

$\Delta R \equiv \sqrt{\left(\eta_{\text {cone }}-\eta_{k_{T}}\right)^{2}+\left(\phi_{\text {cone }}-\phi_{k_{T}}\right)^{2}}<0.1$. With these definitions, Figure 6 shows that the $k_{\mathrm{T}}$ algorithm typically captures less $E_{\mathrm{T}}$ than the cone. We also find that the difference in $E_{\mathrm{T}}$ assignment depends upon the $E_{\mathrm{T}}$ of the cone jet, and that the relative $E_{T}$ captured by the two algorithms depends strongly on the value of $D$.

\section{References}

1. A. Sill, et al: Nucl. Instr. and Meth. A 447, 1 (2000); T. Affolder, et al., FERMILAB-PUB-03/355-E; G. Ascoli, et al., Nucl. Instr. and Meth. A 268, 33 (1988); T. Dorigo, Nucl. Instr. and Meth. A 461, 560 (2001).

2. F. Abe, et al.: Phys. Rev. D 45, 1448 (1992). 


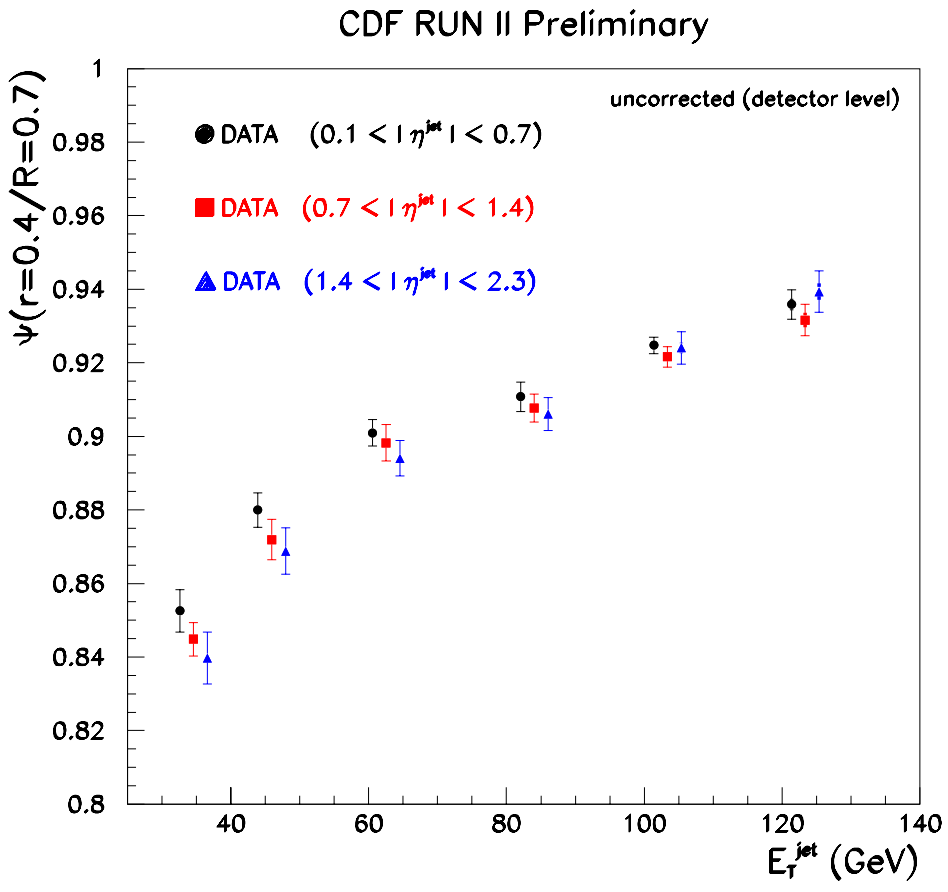

Fig. 5. Measured uncorrected jet shapes, $\Psi(r=0.4)$, as computed using calorimetric information, in different regions of jet $E_{\mathrm{T}}$ and $\eta$. Inner error bars indicate statistical uncertainties, while outer error bars indicate the quadrature sum of statistical and systematic errors.

3. F. Abe, et al.: Phys. Rev. Lett. 77, 438 (1996).

4. J. Pumplin, et al.: JHEP 0207, 012 (2002).

5. J. Bagger, et al.: Phys. Rev. D 37, 1188 (1988).

6. E. Eichten and K. Lane: Phys. Lett. B 327, 129 (1994).

7. U. Baur, et al.: Int. J. Mod. Phys. A2, 1285 (1987); U. Baur, et al.: Phys. Rev. D 42, 815 (1990).

8. G. Katsilieris, et al.: Phys. Lett. B 288, 221 (1992); J. Hewitt and T. Rizzo: Phys. Rep. 183, 193 (1989); V. Angelopoulos, et al.: Nucl. Phys. B 292, 59 (1987).

9. S.D. Ellis and D.E. Soper: Phys. Rev. D 48, 3160 (1993). 


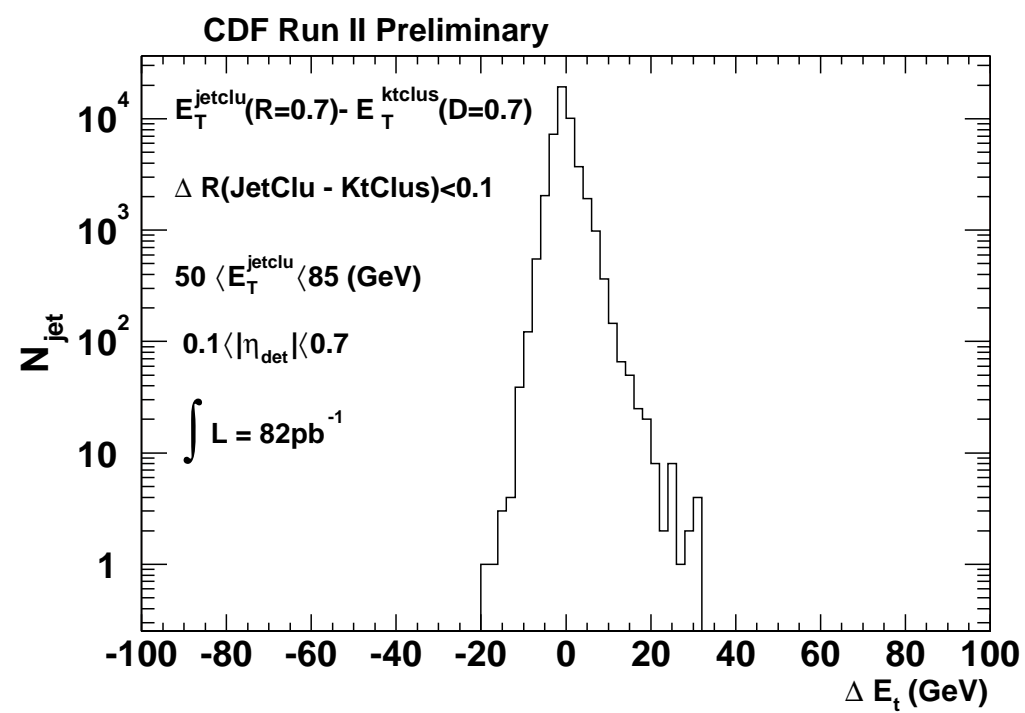

Fig. 6. The difference between the $E_{\mathrm{T}}$ distributions of jets selected with the cone and $k_{\mathrm{T}}$ algorithms, for $R$ and $D$ both equal to 0.7 and $\Delta R<0.1$. 